\title{
CURCUMINA E NORBIXINA: AÇÃO NO METABOLISMO LIPÍDICO DE AVES DOMÉSTICAS
}

\author{
CURCUMIM AND NORBIXIN: ACTION OF LIPIDIC METABOLISM IN POULTRY
}

Rosimar Regina da Silva'; Tania Toledo de Oliveira ${ }^{2}$; Tanus Jorge Nagem ${ }^{3}$; Aloísio da Silva Pinto; Luiz Fernando Teixeira Albino ${ }^{5}$ Márcia Rogéria de Ameida ${ }^{6}$ \& Maria Aparecida Leão ${ }^{7}$

\begin{abstract}
${ }^{1}$ Mestranda em Agroquímica. ${ }^{2}$ Docente. Departamento de Bioquímica e Biologia Molecular-Universidade Federal de Viçosa-MG (UFV) ${ }^{3}$ Docente. Departamento de Química- Universidade Federal de Ouro Preto-MG. ${ }^{4}$ Docente.Departamento de Veterinária-UFV. ${ }^{5}$ Docente. Departamento de Zootecnia-UFV. ${ }^{6}$ Docente. Departamento de Bioquímica e Biologia Molecular-UFV. ${ }^{7}$ Técnico. Departamento de Bioquímica e Biologia Molecular-.UFV.

Correspondência: Tania Toledo de Oliveira-Universidade Federal de Viçosa-MG, Departamento de Bioquímica e Biologia MolecularCEP-36571-000, telefone 0318991429, email-ttoledo@mail.ufv.br
\end{abstract}

SILVA RR; OLIVEIRA TT; NAGEM TJ; PINTO AS; ALBINO LFT; ALMEIDAMR \& LEÃO MA. Curcumina e norbixina: ação no metabolismo lipídico de aves domésticas. Medicina, Ribeirão Preto, 34: 177-182, abr./jun. 2001.

RESUMO: Estudos em animais experimentais têm mostrado que corantes naturais previnem o desenvolvimento de doenças cardiovasculares. O objetivo deste trabalho é avaliar o efeito de curcuma e norbixina na redução dos lipídios sanguíneos em pintos hipercolesterolêmicos. Os resultados mostraram que curcuma e norbixina provocaram reduções significativas nos níveis de colesterol total, colesterol-LDL, colesterol-VLDL e triacilglicerol, não se apresentando reduções nos níveis de colesterol-HDL

UNITERMOS:Curcumina.Norbixina.Colesterol.Aves Domésticas

\section{INTRODUÇÃO}

Estudos epidemiológicos têm estabelecido a importância de fatores dietéticos no desenvolvimento de doenças cardiovasculares em homens e animais, evidenciando uma relação direta entre o nível de lipídios no sangue, principalmente o do colesterol, e o grau de aterogênese ${ }^{(1)}$.

A investigação lipídica é um dos campos mais importantes para o controle da aterosclerose, uma das principais causas de morte no mundo moderno.

O corante extraído do rizoma seco de Curcuma longa $L$., cujo principal princípio ativo é a curcumina, tem sido utilizado no sistema tradicional da medicina indiana. Srinivasan \& Sambaiah, 1991 ${ }^{(2)}$, estudando enzimas retiradas dos tecidos hepáticos de ratos, verificaram efeitos da curcumina sobre o metabolismo dos ácidos biliares e colesterol. A atividade da enzima colesterol-7- $\alpha$-hidroxilase aumentou significativamente em ratos tratados com curcumina. Esta aumentou a excreção fecal de colesterol e ácidos biliares, sendo também um potente eliminador de espécies reativas de oxigênio, protegendo a hemoglobina de oxidação induzida por nitrito, e inibindo a peroxidação lipídica. Muitas dessas atividades são também responsáveis pela sua habilidade de proteger o DNA de danos provo- 
cados por radicais livres ${ }^{(3)}$. Outro mecanismo de ação, pesquisado por Goud et al., $1993^{(4)}$, foi a atividade de enzimas metabolizadoras de xenobióticos em tecidos hepáticos de ratos alimentados com curcuma; observou-se elevação na atividade da enzima UDP glicuronil transferase e da enzima glutationa-S-transferase, sugerindo a ação da curcuma sobre a atividade de enzimas metabolizadoras de drogas.

Conney et al., $1991^{(5)}$, estudaram o efeito inibitório de curcumina e alguns outros compostos da dieta como os ácidos fenólicos (ácido clorogênico, caféico e ferúlico) na promoção de tumor e no metabolismo do ácido araquidônico. Além disso, a curcumina atua como um antiinflamatório, reduzindo a secreção das enzimas colagenase, elastase e hialuronidase em macrófagos ativados ${ }^{(6)}$.

Os carotenóides são pigmentos naturais, responsáveis pela coloração amarela, laranja e vermelha de grande número de frutas, folhas e algumas flores ${ }^{(7)}$. Dentre os carotenóides estão o alfa, beta e gamacaroteno, bixina, norbixina, capsantina, capsorubina e licopeno. O corante de urucum de coloração laranja avermelhado é extraído de sementes de espécie Bixa orellana $L$, e a bixina é o principal pigmento. A bixina pode ser saponificada, formando um corante hidromiscível constituído pelo sal de norbixina. Ambas, bixina e norbixina, podem estar presentes em uma extração e podem também existir nas formas cis e trans. Existe uma relação inversa entre o risco de câncer e hiperlipidemias e o consumo de alimentos contendo carotenóides, demonstrando-se ação anticancerígena e hipolipidêmica ${ }^{(8)}$.

Os carotenóides possuem, também, atividade antioxidante e podem atuar sinergicamente com outros antioxidantes, proporcionando uma maior proteção contra espécies reativas de oxigênio. Esta ação é importante, uma vez que a LDL quando oxidada danifica o endotélio e inicia o processo de aterosclerose ${ }^{(9)}$.

O presente trabalho teve como objetivo avaliar os possíveis efeitos dos corantes naturais curcuma e norbixina no metabolismo lipídico de aves domésticas hiperlipidêmicas.

\section{MATERIAL E MÉTODOS}

Para a realização do ensaio biológico foram utilizadas aves da linhagem Avian farms, (pintos de corte) machos, com sete dias de vida, que foram distri- buídos em quatro grupos contendo em cada grupo 10 animais, totalizando 40 animais, provenientes do Departamento de Zootecnia da Universidade Federal de Viçosa, com peso médio de $150 \mathrm{~g}$, e que receberam ração e água ad libitum durante 28 dias. $\mathrm{O}$ experimento foi delineado de forma inteiramente casualizada, com quatro tratamentos, em 10 repetições:

GRUPO 1 (G1): Ração, GRUPO 2 (G2): Ração $+10 \%$ de gordura de suíno $+0,7 \%$ de colesterol + 0,1\% de ácido cólico, GRUPO 3 (G3): Ração + 10\% de gordura de suíno $+0,7 \%$ de colesterol $+0,1 \%$ de ácido cólico + 20mg de norbixina, GRUPO 4 (G4): Ração $+10 \%$ de gordura de suíno $+0,7 \%$ de colesterol $+0,1 \%$ de ácido cólico $+20 \mathrm{mg}$ de curcuma.

Os corantes naturais norbixina e curcuma foram fornecidos pela indústria Christian Hansen. e administrados via dieta, misturados à ração, diariamente, na proporção de $20 \mathrm{mg} / \mathrm{ave} / \mathrm{dia}$.

As amostras de sangue das aves foram coletadas por punção cardíaca no $7^{0}, 14^{0}, 21^{0}$ e $28^{0}$ dias, após jejum de 12 horas, centrifugadas a 3050 x g e colesterol total, colesterol-lipoproteína de baixa densidade (colesterol-LDL), colesterol-lipoproteína de alta densidade (colesterol-HDL), colesterol-lipoproteína de muito baixa densidade (colesterol-VLDL) e triacilglicerol, foram dosados no soro utilizando-se equipamento Alizé (Analisador automático de bioquímica ), e kits da marca Biomérieux.Os resultados foram expressos em $\mathrm{mg} / \mathrm{dL}$.

A análise colorimétrica do colesterol total, utilizando-se o método enzimático de colesterol-oxidase, e de colesterol-HDL bem como colesterol -LDL além da dosagem dos triacilgliceróis séricos, realizada utilizando-se o método enzimático com reações sequiênciais com as enzimas glicerolquinase, glicerofosfatoxidase e peroxidase foi feita de acordo com Carl \& Ashwood, 1994 ${ }^{(10)}$.

O percentual de variação média dos parâmetros avaliados foi calculado em relação aos controles G1 e G2, aos $14^{\circ}, 21^{\circ}$ e $28^{\circ}$ dias, individualmente.

Para a análise estatística, procedeu-se à análise de variância e teste $\mathrm{F}(\mathrm{P}<0,05$ e $\mathrm{P}<0,01)$ para as características mensuradas, considerando os grupos G1 e G2 como controles. Esses controles foram comparados entre si pelo teste F. Da mesma forma foi feita a comparação dos grupos G3 e G4. O efeito dos corantes foi analisado por meio do teste de Dunnett, a $5 \%$ de probabilidade. 


\section{RESULTADOS}

Os resultados das médias das concentrações de colesterol total, colesterol-HDL, colesterol-LDL, colesterol-VLDL e triacilgliceróis estão mostrados nas Tabelas I, III $\mathrm{V}$, VII e IX respectivamente, expressos em $\mathrm{mg} / \mathrm{dL}$, com suas respectivas percentagens de variações inscritas nas Tabelas II, IV, VIII, X.

Pela Tabela I, pode-se observar que os animais do grupo 2, que receberam gordura de suíno, colesterol e ácido cólico na dieta, apresentaram aumento significativo nos níveis de colesterol total durante todo o período experimental (21 dias), quando comparados com o grupo 1, comprovandose o efeito hipercolesterolêmico daquelas substâncias.

Comparando-se os grupos 3 e 4, notase que apenas no $28^{\circ}$ dia houve diferença significativa entre os tratamentos, embora ambos tenham provocado reduções significativas nos níveis de colesterol total. Os melhores resultados foram observados no grupo 4 , tratados com curcuma, cujas percentagens de redução foram de $28 \%$ no $21^{\circ}$ dia e de $57 \%$ no $28^{\circ}$ dia, o que demonstram o efeito hipocolesterolêmico dos corantes naturais, testados, em aves com hiperlipidemia induzida pela dieta.

Quanto aos níveis de colesterol-HDL e respectivas percentagens de variação, os resultados mostram que tanto o grupo 3 quanto o grupo 4, não apresentaram diferenças significativas, quando comparados com o grupo 2. Tal fato é importante, tendo em vista que o colesterol-HDL previne a formação de placas ateroscleróticas e é responsável pelo transporte do colesterol dos tecidos periféricos para o fígado ${ }^{(11)}$. Já os níveis mais elevados do grupo 2 podem indicar um elevado transporte reverso do colesterol dos tecidos periféricos para o fígado, onde ocorre catabolismo e excreção do colesterol.

Quanto aos níveis de colesterol-LDL e respectivas percentagens de variação, os resultados mostram que ambos os tratamentos, em todos os períodos, resultaram em reduções significativas, sendo que o grupo 4
Tabela I: Colesterol total médio, em $\mathrm{mg} / \mathrm{dL}$, de aves submetidas a diferentes tratamentos e avaliadas durante 28 dias

\begin{tabular}{ccccc} 
Grupos & \multicolumn{4}{c}{ Tempo (dias) } \\
\cline { 2 - 5 } Tratados & 7 & 14 & 21 & 28 \\
G1 & 145,50 & $143,83 \mathrm{~B}$ & $131,17 \mathrm{~B}$ & $141,67 \mathrm{~B}$ \\
G2 & 145,50 & $232,67 \mathrm{~A}$ & $291,33 \mathrm{~A}$ & $338,83 \mathrm{~A}$ \\
G3 & 145,50 & $212,00 \mathrm{a}$ & $242,17 \mathrm{a}$ & $271,83 \mathrm{a}$ \\
G4 & 145,50 & $229,17 \mathrm{a}$ & $209,00 \mathrm{a}$ & $143,83 \mathrm{~b}$ \\
\hline
\end{tabular}

Em cada tempo, A difere de $B$ pelo teste $F(P<0,05)$, na comparação de G1 com G2.

Em cada tempo, a difere de $b$ pelo teste $F(P<0,05)$, na comparação de $G 3$ com G4.

Tabela II: Percentual de variação em relação aos controles G1 e G2, do colesterol total médio de aves submetidas a diferentes tratamentos e avaliadas durante 28 dias

\begin{tabular}{ccccc} 
Em relação & Grupos & \multicolumn{3}{c}{ Tempo (dias) } \\
\cline { 3 - 5 } ao Controle & tratados & 14 & 21 & 28 \\
& G3 & $+47,40 *$ & $+84,62^{*}$ & $+91,88^{*}$ \\
& G4 & $+59,33^{*}$ & $+59,34^{*}$ & $+1,52$ \\
\hline \multirow{2}{*}{ G2 } & G3 & $-8,88$ & $-16,87^{*}$ & $-19,77^{*}$ \\
& G4 & $-1,50$ & $-28,26$ * & $-57,55^{*}$
\end{tabular}

Estatisticamente diferente do controle pelo teste de Dunnett $(P<0,05)$.

Tabela III: Colestrol-HDL médio, em $\mathrm{mg} / \mathrm{dL}$, de aves submetidas a diferentes tratamentos e avaliadas durante 28 dias

\begin{tabular}{ccccc} 
Grupos & \multicolumn{4}{c}{ Tempo (dias) } \\
\cline { 2 - 5 } Tratados & 7 & 14 & 21 & 28 \\
G1 & 64,50 & $102,67 \mathrm{~A}$ & $87,00 \mathrm{~A}$ & $102,17 \mathrm{~A}$ \\
G2 & 64,50 & $105,17 \mathrm{~A}$ & $101,67 \mathrm{~A}$ & $84,33 \mathrm{~B}$ \\
G3 & 64,50 & $100,67 \mathrm{a}$ & $104,67 \mathrm{a}$ & $83,17 \mathrm{a}$ \\
G4 & 64,50 & $103,83 \mathrm{a}$ & $121,67 \mathrm{a}$ & $83,83 \mathrm{a}$
\end{tabular}

Em cada tempo, A difere de $B$ pelo teste $F(P<0,05)$, na comparação de G1 com G2.

Em cada tempo, a difere de b pelo teste $F(P<0,05)$, na comparação de $G 3$ com G4.

Tabela IV: Percentual de variação em relação aos controles G1 e G2, do colesterol-HDL médio de aves submetidas a diferentes tratamentos e avaliadas durante 28 dias

\begin{tabular}{ccccc}
\hline \multirow{2}{*}{$\begin{array}{c}\text { Em } \\
\text { relação a: }\end{array}$} & $\begin{array}{c}\text { Grupos } \\
\text { tratados }\end{array}$ & \multicolumn{3}{c}{ Tempo (dias) } \\
\cline { 3 - 5 } G1 & G3 & $-1,95$ & $+20,31$ & $-18,60$ * \\
& G4 & $+1,13$ & $+39,85^{*}$ & $-17,95^{*}$ \\
\hline \multirow{2}{*}{ G2 } & G3 & $-4,28$ & $+2,95$ & $-1,38$ \\
& $G 4$ & $-1,27$ & $+19,67$ & $-0,59$ \\
\hline
\end{tabular}

Estatisticamente diferente do controle pelo teste de Dunnett $(P<0,05)$. 
Tabela V: Colesterol-LDL médio, em $\mathrm{mg} / \mathrm{dL}$, de aves submetidas a diferentes tratamentos e avaliadas durante 28 dias

\begin{tabular}{ccrrr} 
Grupos & \multicolumn{4}{c}{ Tempo (dias) } \\
\cline { 2 - 5 } Tratados & 7 & 14 & 21 & 28 \\
G1 & 29,50 & $21,17 \mathrm{~B}$ & $24,00 \mathrm{~B}$ & $26,83 \mathrm{~B}$ \\
G2 & 29,50 & $113,83 \mathrm{~A}$ & $145,00 \mathrm{~A}$ & $213,00 \mathrm{~A}$ \\
G3 & 29,50 & $90,17 \mathrm{a}$ & $140,50 \mathrm{a}$ & $180,67 \mathrm{a}$ \\
G4 & 29,50 & $103,17 \mathrm{a}$ & $86,83 \mathrm{~b}$ & $64,17 \mathrm{~b}$ \\
\hline
\end{tabular}

Em cada tempo, A difere de $B$ pelo teste $F(P<0,05)$, na comparação de G1 com G2.

Em cada tempo, a difere de $b$ pelo teste $F(P<0,05)$, na comparação de $G 3$ com G4.

Tabela VI: Percentual de variação em relação aos controles G1 e G2, do colesterol-LDL médio de aves submetidas a diferentes tratamentos e avaliadas durante 28 dias

\begin{tabular}{ccccc} 
Em relação & Grupos & \multicolumn{3}{c}{ Tempo (dias) } \\
\cline { 3 - 5 } a: & tratados & 14 & 21 & 28 \\
G1 & G3 & $+325,93^{*}$ & $+485,42 *$ & $+573,39$ * \\
& G4 & $+387,34^{*}$ & $+261,79 *$ & $+139,17$ \\
\hline \multirow{2}{*}{ G2 } & G3 & $-20,79$ & $-3,10$ & $-15,18$ \\
& G4 & $-9,36$ & $-40,12 *$ & $-69,87$ *
\end{tabular}

Estatisticamente diferente do controle pelo teste de Dunnett $(\mathrm{P}<0,05)$.

Tabela VII: Colesterol-VLDL médio, em $\mathrm{mg} / \mathrm{dL}$, de aves submetidas a diferentes tratamentos e avaliadas durante 28 dias

\begin{tabular}{ccrrr|} 
Grupos & \multicolumn{4}{c|}{ Tempo (dias) } \\
\cline { 2 - 5 } Tratados & 7 & 14 & 21 & 28 \\
G1 & 7,57 & $6,57 \mathrm{~B}$ & $5,80 \mathrm{~B}$ & $4,93 \mathrm{~B}$ \\
G2 & 7,57 & $32,17 \mathrm{~A}$ & $48,53 \mathrm{~A}$ & $48,90 \mathrm{~A}$ \\
G3 & 7,57 & $5,20 \mathrm{a}$ & $5,40 \mathrm{a}$ & $5,17 \mathrm{a}$ \\
G4 & 7,57 & $5,57 \mathrm{a}$ & $5,43 \mathrm{a}$ & $3,87 \mathrm{a}$ \\
\hline
\end{tabular}

Em cada tempo, A difere de $B$ pelo teste $F(P<0,05)$, na comparação de G1 com G2.

Em cada tempo, a difere de b pelo teste $F(P<0,05)$, na comparação de $G 3$ com G4.

Tabela VIII: Percentual de variação em relação aos controles G1 e G2, do Colesterol-VLDL médio de aves submetidas a diferentes tratamentos e avaliadas durante 28 dias

\begin{tabular}{ccccc}
\hline \multirow{2}{*}{$\begin{array}{c}\text { Em relação } \\
\text { a: }\end{array}$} & $\begin{array}{c}\text { Grupos } \\
\text { tratados }\end{array}$ & \multicolumn{3}{c}{ Tempo (dias) } \\
\cline { 3 - 5 } G1 & G3 & $-20,85$ & $-6,90$ & $+4,87$ \\
& G4 & $-15,22$ & $-6,38$ & $-21,50$ \\
\hline \multirow{2}{*}{ G2 } & G3 & $-83,84$ * & $-88,87$ * & $-89,43$ * \\
& G4 & $-82,69$ * & $-88,81$ * & $-92,09$ *
\end{tabular}

* Estatisticamente diferente do controle pelo teste de Dunnett $(P<0,05)$. apresentou maior nível de redução (69\%). A redução dos níveis de colesterol-LDL está diretamente relacionada com redução de doenças cardiovasculares, uma vez que as citadas lipoproteínas, quando acumuladas, levam ao processo da aterosclerose, pois danificam o endotélio, quando oxida$\operatorname{das}^{(12)}$.

Com relação aos níveis de colesterolVLDL, os resultados obtidos mostram que tanto o grupo 3 quanto o grupo 4 apresentaram reduções elevadas nos níveis sanguíneos desta lipoproteína, que é transportadora de triacilgliceróis.

Os níveis de triacilgliceróis mostram que tanto a norbixina quanto a curcuma apresentaram níveis significativos de redução ( $89 \%$ e $92 \%$, respectivamente), quando comparados com o grupo 2.

\section{DISCUSSÃO}

Aves têm sido considerado como um modelo animal, adequado para a bioquímica comparativa do metabolismo e transporte de colesterol $^{(1)}$.

Subba Rao et al., $1970^{(13)}$, em estudos com animais experimentais têm mostrado que curcuma apresenta efeito hipolipidêmico envolvendo vários mecanismos de ação, sendo um deles aumentar a excreção fecal de sais biliares e colesterol em ratos hiperlipidêmicos.

Segundo Engelberg, 1956(14) curcumina aumenta a atividade da enzima $7 \alpha$-hidroxilase, o que leva a uma maior conversão de colesterol em sais biliares, reduzindo assim os níveis sanguíneos de colesterol. Estudos de Morin et al., 1962 ${ }^{(15)}$, mostraram um aumento na deposição de lipídios no fígado, em presença de curcumina, colesterol e óleo de coco, em animais que receberam tal tratamento na dieta.

Suresh Babu \& Srinivasan, 1995(16), relatam efeito hipocolesterolêmico de curcumina em ratos com hiperlipidemia. Pulla Reddy et al., 1992 ${ }^{(17)}$, relatam, também, as ações de curcumina como antioxidantes e inibidoras da peroxidação lipídica em microssomos de fígado de ratos. Esta atividade 
Tabela IX: Triacilglicerol médio, em $\mathrm{mg} / \mathrm{dL}$, de aves submetidas a diferentes tratamentos e avaliadas durante 28 dias

\begin{tabular}{ccrrr} 
Grupos & \multicolumn{4}{c}{ Tempo (dias) } \\
\cline { 2 - 5 } Tratados & 7 & 14 & 21 & \multicolumn{1}{c}{28} \\
G1 & 37,83 & $32,83 \mathrm{~B}$ & $29,00 \mathrm{~B}$ & $24,67 \mathrm{~B}$ \\
G2 & 37,83 & $160,83 \mathrm{~A}$ & $240,17 \mathrm{~A}$ & $244,50 \mathrm{~A}$ \\
G3 & 37,83 & $26,00 \mathrm{a}$ & $27,00 \mathrm{a}$ & $25,83 \mathrm{a}$ \\
G4 & 37,83 & $27,83 \mathrm{a}$ & $27,17 \mathrm{a}$ & $19,33 \mathrm{a}$ \\
\hline
\end{tabular}

Em cada tempo, $A$ difere de $B$ pelo teste $F(P<0,05)$, na comparação de G1 com G2.

Em cada tempo, a difere de b pelo teste $F(P<0,05)$, na comparação de $G 3$ com G4.

Tabela X: Percentual de variação em relação aos controles G1 e G2, do triacilglicerol médio de aves submetidas a diferentes tratamentos e avaliadas durante 28 dias

\begin{tabular}{|ccccc|}
\hline \multirow{2}{*}{$\begin{array}{c}\text { Em relação } \\
\text { a: }\end{array}$} & $\begin{array}{c}\text { Grupos } \\
\text { tratados }\end{array}$ & 14 & 21 & 28 \\
\cline { 3 - 5 } G1 & G3 & $-20,80$ & $-6,90$ & $+4,70$ \\
& G4 & $-15,23$ & $-6,31$ & $-21,65$ \\
\hline \multirow{2}{*}{ G2 } & G3 & $-83,83$ * & $-88,76$ * & $-89,44$ * \\
& G4 & $-82,70$ * & $-88,69$ * & $-92,09$ * \\
\hline \multirow{2}{*}{ * Estatisticamente diferente do controle pelo teste de Dunnett $(P<0,05)}$. \\
\hline
\end{tabular}

A literatura ${ }^{(20)}$ relata que, em pintos recém-eclodidos, o depósito de colesterol no músculo esquelético estimula a síntese de apoproteína A-I, que é a apoproteína característica da HDL. Essa apoproteína ativa a enzima LCAT, que esterifica o colesterol presente na superfície da HDL, que converte o colesterol presente em quilomícrons, VLDL, LDL e tecidos periféricos em ésteres de colesterol, para serem transportados para o fígado. Quando pintos recém-eclodidos são alimentados com dieta aterogênica, a síntese de apoproteína A-1 e HDL pode ser mantida por mais tempo, o que se verifica quando são comparados com animais alimentados com dietas normais. $\mathrm{O}$ aumento de HDL pode ser causado pelo acúmulo de colesterol nos tecidos.

\section{CONCLUSÃO}

Os resultados obtidos permitem concluir que curcuma e norbixina induzem reduções significativas nos níveis de colesterol total, colesterol-LDL, colesterol-VLDL e triacilglicerol. Em relação aos níveis de

impede a oxidação do LDL. Elizabeth \& Rao, $1990^{(18)}$, Unnikrishnan \& Rao, $1992^{(19)}$, relatam os efeitos de curcumina como antiinflamatórios inibindo a formação de prostaglandinas e leucotrienos. Nesse mecanismo de ação ocorre a inibição das enzimas de ciclooxigenase e lipoxigenase, que são responsáveis pela formação dessas substâncias em processos inflamatórios da aterosclerose.
colesterol-HDL, observa-se que não houve variação quando os grupos 3 e 4 foram comparados com o grupo 2, fato importante uma vez que tais lipoproteínas são responsáveis pelo transporte do excesso de colesterol dos tecidos periféricos para o fígado, justificando-se assim, a utilização de medidas e fármacos para elevar os níveis de HDL, com a finalidade de preservar a função endotelial e prevenir a aterogênese.

SILVA RR; OLIVEIRA TT; NAGEM TJ; PINTO AS; ALBINO LFT; ALMEIDA MR \& LEÃO MA. Curcumim and norbixin: action of lipidic metabolism in poultry. Medicina, Ribeirão Preto, 34: 177-182, april/june. 2001.

ABSTRACT: Studies in experimental animals have been suggesting that natural colorants they protect against the development of cardiovascular diseases. The objective went evaluate the curcumin effect and norbixin in the reduction of the blood lipids in poultry hypercholesterolemics. The results showed that curcumin and norbixin presented significant reductions in the levels of total cholesterol, cholesterol-LDL, cholesterol-VLDL and triacylglicerol, not presenting reductions in the levels of cholesterol-HDL.

UNITERMS: Curcumin. Norbixin. Cholesterol. Poultry. 


\section{REFERÊNCIAS BIBLIOGRÁFICAS}

1 - CASTILLO M; HORTAL JH; AGUILERA JA; ZAFRA MF \& CARCIA-PEREGRIN E. Different hypercholesterolemic effects of cholesterol and saturated fat on neonatal and adult chicks. Comp Biochem. Physiol 107A:.209-213, 1994.

2 - SRINIVASAN K \& SAMBAIAH K. The effect of spices on cholesterol levels 7a-Hidroxilase activity and on serum e hepatic cholesterol levels in the rat. Int J Vitam Nutr Res 61: 364369, 1991.

3 - REDDY S \& AGGARWAL BB Curcumin is a non-competitive and selective inhibitor of phosphorylase kinase. FEBS Lett 341: 19-22, 1994.

4 - GOUD V K; POLASA K \& KRISHNASWAMY K. Effect of turmeric on xenobiotic metabolising enzymes. Plant Foods Hum Nutr 44: 87-92, 1993.

5 - CONNEY AH; LYSZ T; FERRARO T; ABIDI TF; MANCHAND PS; LASKIN JD \& HUANG MT Inhibitory effects of curcumin and some related dietary comprounds on tumor promotion and arachidonic acid metabolism in mouse skin. Adv Enzyme Regul 31: 385-396, 1991.

6 - JOE B \& LOKESH B R. Effect of curcumin and capsaicin on arachidonic acid metabolism and lysosomal enzyme secretion by rat peritoneal macrophages. Lipids 32: 11731180, 1997.

7 - BOBBIO P A \& BOBBIO FO. Química de processamento de alimentos. $2^{3}$ ed, Varela, São Paulo, 1992. 223p.

8 - KHACHIK F; ASKIN F B \& LAI K. Distribution, bioavailability, and metabolism of carotenoids in humans. In: BIDLACK WR; OMAYE ST; MESKIN M S \& JAHNER D. Phytochemicals: A new paradigm. Lancaster Basel, Pennsylvania, p 80-95, 1998.

9 - STAHL W \& SIES H Lycopene: a biologically important carotenoid for humans. Arch Biochem Biophys 336: 1-9, 1996.

10 - CARL AB \& ASHWOOD ER. Tietz Texbook of clinical chemistry, 2 ed. W.B.Saunders, Philadelphia, cap. 23 , p. 10551080, 1994.
11 - MAHAN K L \& ARLIN M T. Nutrição na doença aterosclerótica cardiovascular. In: MAHAN K L \& ARLIN M T. Alimentos, nutrição e dietoterapia. $8^{\mathrm{a}}$ ed., Editora Roca, São Paulo, cap 20, p.377-408, 1995.

12 - STEINBERG D; PATHASARATHY S; CAREW TE; KHOO J C \& WITZUM J L. Modification of low density lipoprotein that increases its atherogenicity. J Med 320: 915-924, 1989.

13 - SUBBA RAO D; CHANDRA SEKHARA N; SATYANARAYANA $M N$ \& SRINIVASAN M. Effect of curcumin on serum and liver cholesterol levels in the rat. J Nutr 100: 1307-1316, 1970.

14 - ENGELBERG H. Short term studies on the effect of heparin on cholesterol excretion in man. Proc Soc Exp Biol Med 102: 365-378, 1956.

15 - MORIN R J; BERNICK J F; MEAD J F \& ALFIN-SLATER R B. The influence of exogenous cholesterol on hepatic liver composition of the rat. J Lipid Res 3: 432-443, 1962.

16 - SURESH BABU PS \& SRINIVASAN K. Influence of dietary curcumin and cholesterol on the progression of experimentally induced diabetes in albino rat. Mol Cell Biochem 152: 13-21, 1995.

17 - PULLA REDDY A \& LOKESH BR. Studies on spice principles as antioxidants in the inhibition of lipid peroxidation of rat liver microsomes. Mol Cell Biochem, 111: 117-124, 1992.

18 - ELIZABETH K \& RAO MNA. Oxygen radical scavenging activity of curcumin. Int J Pharmacol 58: 237-240, 1990.

19 - UNNIKRISHNAN MBS \& RAO MNA. Curcumin inhibits nitrate induced methaemoglobin formation. FEBS Lett 301: 195196, 1992.

20 - JIANG Z; CHERIAN G; ROBINSON FE \& SIM JS. Effect of feeding cholesterol to laying hens and chicks on cholesterol metabolism in pre- and posthatch chicks. Poult Sci 69:16941701, 1990.

Recebido para publicação em: 06/09/2000

Aprovado para publicação em 26/06/2001 